\title{
Tamanho Ótimo do Pacote em Comunicação por Luz Visível Sem Fio
}

\author{
André G. Vieira ${ }^{1}$, Luiz F. M. Vieira ${ }^{1}$, Marcos A. M. Vieira ${ }^{1}$ \\ ${ }^{1}$ Departamento de Ciência da computação \\ Universidade Federal de Minas Gerais (UFMG) \\ CEP: 31270-901 - Belo Horizonte - MG - Brasil \\ \{andregarcia, lfvieira, mmvieira\}edcc.ufmg.br
}

\begin{abstract}
Optical Wireless Communication (OWC) has shown to be one of the complementary technologies to radio-frequency, which can solve the lack of spectrum. Furthermore, new applications such as Internet of Things and wireless sensor networks can benefit from OWC. With the increase of devices connected, the efficiency of channel usage becomes necessary. This paper presents a channel modeling and develops a method to define the efficiency of channel usage. The model was validated experimentally with the OpenVLC 1.1 platform. With it, it is possible to define the optimal packet size to maximize the efficiency of channel usage. The correlation between the experimental and model data was up to 0,998 .
\end{abstract}

Resumo. A comunicação óptica sem fio $(O W C)$ tem se mostrado como uma das tecnologias de comunicação complementares à radiofrequência que pode solucionar o problema de falta de espectro. Além disso, novas aplicações como Internet das Coisas e rede sensores sem fio podem se beneficiar do OWC. Com o aumento de dispositivos conectados, a eficiência de uso do canal se torna imprescindivel. Este artigo apresenta a modelagem do canal e desenvolve um método para determinar a eficiência do uso do canal. O modelo foi validado experimentalmente com a plataforma OpenVLC 1.1. Com ele, é possível determinar o tamanho ótimo do pacote para maximizar a eficiência do uso canal. A correlação entre os dados experimentais e os dados do modelo foi de até 0,998.

\section{Introdução}

A Comunicação óptica sem fio (OWC - do inglês Optical Wireless Communication) vem ganhando destaque tanto por parte da indústria quanto da academia. Ela tem se mostrado como uma alternativa complementar a radiofrequência e pode solucionar a lotação do espectro. Essa ocupação da banda tem chamado atenção da academia [Vries et al. 2014]. De fato, o número de dispositivos móveis conectados à Internet vêm aumentado e isso leva a uma demanda maior por largura de banda e uma procura por tecnologias alternativas.

A Cisco [CISCO 2019] dimensiona que até 2030, 500 bilhões de dispositivos estarão conectados à Internet. Todos esses dispositivos demandarão largura de banda e gerarão muito tráfego. O OWC surge como uma tecnologia de comunicação promissora capaz de fornecer os parâmetros necessários para conectividade que os novos dispositivos demandam. A concorrência e demanda pelo uso do canal serão grandes. Portanto, o uso do canal óptico sem fio deve ser feito com a maior eficiência possível. 
Existem diversas aplicações que podem se beneficiar e fazer uso da comunicação óptica sem fio. O OWC pode ser aplicado tanto em demandas civis quanto em sistemas militares [Ucar et al. 2016]. Vários sistemas podem ter seus parâmetros de conexão aprimorados com o uso da comunicação óptica, como em sistemas em tempo real [Zuquim et al. 2003], em redes de comunicação veículo-veículo (V2V) ou em redes industriais com comunicação máquina-máquina (M2M).

O uso de um sistema de comunicação óptica sem fio pode trazer vantagens quando comparado a sistemas de radiofrequência tradicionais graças ao fato de a luz não atravessar paredes. Uma rede interna utilizando o canal óptico sem fio não corre o risco de algum dispositivo fora do ambiente capturar os dados. Um outro fator reside no fato de que a infraestrutura de iluminação já está instalada e o OWC pode fazer uso dessas instalações [Pathak et al. 2015].

As principais contribuições desse artigo são: (i) apresentação de uma modelagem para o canal de comunicação óptica sem fio, (ii) desenvolvimento de um modelo de maximização da eficiência do uso do canal em OWC, (iii) validação experimental do modelo de eficiência e (iv) resultados que mostram correlação entre dados experimentais e o modelo de até $99,8 \%$.

O restante do artigo está organizado da seguinte maneira: na seção 2 são descritos os trabalhos que se relacionam com o tema deste artigo. A seção 3 apresenta a comunicação óptica sem fio e traz um modelo do canal. A seção 4 modela o problema e introduz os resultados obtidos com tal modelagem. Já a seção 5 faz uma avaliação experimental de tal modelo. Finalmente, na seção 6 é feita a conclusão e são indicados trabalhos futuros.

\section{Trabalhos Relacionados}

Aqui, são descritos os trabalhos relacionados com o tamanho ótimo do pacote, com a maximização da eficiência do uso do canal e com comunicação óptica sem fio.

Pompili et al. [Pompili et al. 2006] fazem uma correlação entre eficiência do uso do canal e o tamanho de pacote que otimiza essa eficiência para redes aquáticas. Soares Junior e Vieira [Soares Junior and Vieira 2014] mostram uma correlação entre o tamanho do pacote e a eficiência do uso do canal. Eles otimizaram o tamanho do pacote de maneira a otimizar o uso do canal. Contudo, os autores fizeram para o uso em redes ZigBee [Alliance 2004].

Alguns trabalhos discutiram a otimização de parâmetros de rede utilizando o tamanho do pacote como variável de decisão. Sankarasubramaniam et al. [Sankarasubramaniam et al. 2003] discutem a otimização do tamanho do pacote com foco em eficiência energética para redes sensores sem fio. Entretanto, Jun Yin et al. [Jun Yin et al. 2004] fazem uma modelagem do tamanho ótimo do pacote com o objetivo de maximizar a vazão. Já Vieira et al. [Vieira et al. 2018] propõem um protocolo que reduz a latência de transmissões OWC utilizando codificação de rede. Entretanto, nenhum trabalho que descreva a eficiência do uso do canal óptico sem fio foi encontrado.

A comunicação por luz visível (VLC) e a comunicação óptica sem fio tem aumentado o número de pesquisas e as publicações crescem a cada ano [Matheus et al. 2019]. Shao et al. [Shao et al. 2014] e Basnayaka e Haas [Basnayaka and Haas 2015] propõe o 
uso de sistemas híbridos de comunicação que utilizam RF e VLC de maneira complementares.

Em cenários de rede $A d$ Hoc Cen et al. [Cen et al. 2019] citam diversas aplicações que podem utilizar-se da tecnologia VLC, seja com objetivos militares ou civis. Dentre os pontos abordados pelo trabalho podemos destacar a alta segurança de redes OWC e o baixo consumo de energia quando comparado a algumas tecnologias de radiofrequência.

Em roteamento, Jagannath e Melodia [Jagannath and Melodia 2019] desenvolveram um protocolo de roteamento para OWC. Tal protocolo faz uso de informações da camada de enlace para realizar o roteamento. Matheus et al. [Matheus et al. 2018] também desenvolveram um protocolo de roteamento dinâmico, para OWC, que é capaz de se adaptar as mudanças na topologia de rede ou até mesmo efeitos de sombra e obstáculos.

Sumarizando, existem diversos trabalhos na literatura que otimizam o tamanho do pacote para beneficiar algum parâmetro de rede. Porém, nenhum deles otimiza o uso do canal óptico sem fio. Exitem, também, diversos trabalhos sobre comunicação óptica sem fio. Entretanto nenhum deles faz qualquer tipo de otimização do canal. Ao contrário dos demais trabalhos, esse maximiza a eficiência do uso do canal de comunicação óptico através do tamanho ótimo do pacote e apresenta resultados experimentais.

\section{Comunicação óptica sem fio}

O VLC utiliza a faixa do espectro eletromagnético com comprimentos de onda que podem variar entre $380 \mathrm{~nm}$ até $780 \mathrm{~nm}$ e teve seu padrão definido em 2011 [IEEE 2011]. O padrão foi revisado em 2018 [IEEE 2019] trazendo um aumento de cobertura com comprimentos de ondas de $190 \mathrm{~nm}$ até $10000 \mathrm{~nm}$, conforme mostra a figura 1. Essa faixa do espectro inclui frequências desde infravermelho até ultravioleta. $\mathrm{O}$ aumento da faixa utilizada trouxe um novo nome, comunicação óptica sem fio. Portanto, a comunicação por luz visível é um subconjunto da comunicação óptica sem fio.

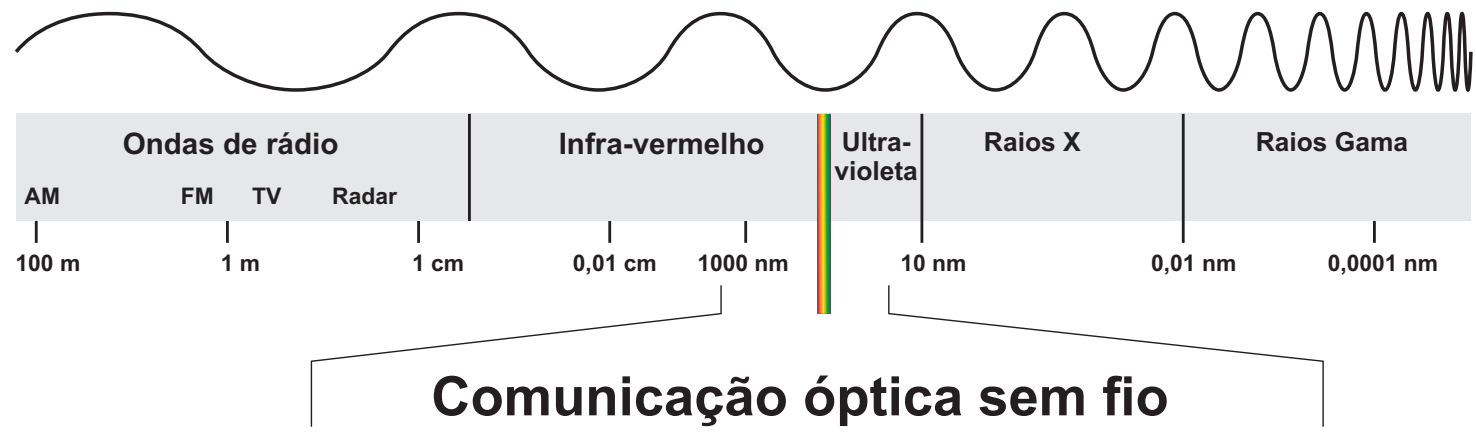

Figura 1. Espectro Eletromagnético.

A luz pode ser usada como meio de comunicação [Matheus et al. 2017] e possui várias áreas de pesquisa, entre elas redes corporais, comunicação entre veículos, cidades inteligentes etc. Há diversas aplicações, incluindo sistema de posicionamento por luz [Kavehrad and Zhang 2015], localização interna [Hassan et al. 2015] e a própria comunicação led-led ou led-fotoreceptor. 


\subsection{Modelo de canal óptico}

A modelagem de canal óptico a ser considerado nesse trabalho é o mesmo descrito por Zhong and Wang [Zhong and Wang 2015]. A potência recebida no fotorreceptor $P_{R}$ é dada pela equação 1 , onde $H(0)$ é a atenuação do canal óptico e $P_{t}$ é a potência de emitida pelo led transmissor. A figura 2 mostra de maneira esquemática dois dispositivos se comunicando e usando o canal óptico sem fio.

$$
P_{R}=H(0) P_{t}
$$

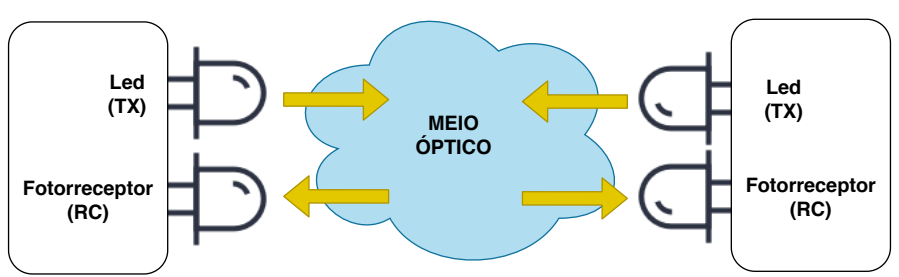

Figura 2. Representação do canal de comunicação óptica.

A figura 3 mostra a geometria que envolve a comunicação entre um led transmitindo dados e um fotorreceptor recebendo dados. Os vetores $\overrightarrow{D_{r}}$ e $\overrightarrow{D_{s}}$ são, respectivamente, vetor normal à superfície do emissor e vetor normal à superfície do receptor. $\mathrm{O}$ vetor $\overrightarrow{D_{r s}}$ é o vetor que aponta do centro do fotorreceptor em direção ao centro do led transmissor.

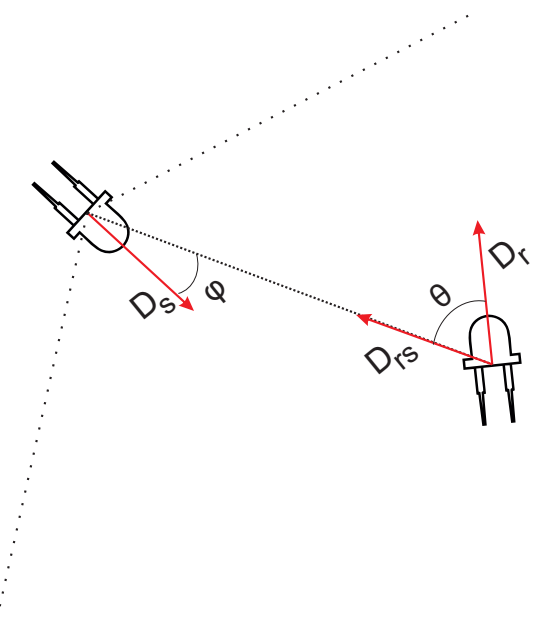

Figura 3. Esquema geométrico óptico entre o emissor e o receptor.

Assumindo que o led emita obedecendo o modelo de emissão Lambertiano, temos que a radiação espectral emitida $\left(R_{(\varphi)}\right)$ pode ser obtida pela equação 2 sendo $m=\frac{\ln 0.5}{\ln \left(\cos \varphi_{1 / 2}\right)}$, onde $\varphi_{1 / 2}$ é o ângulo de meia potência e para o led $\varphi_{1 / 2}=60^{\circ}$. 


$$
R_{(\varphi)}=\frac{(m+1) \cos ^{m} \varphi}{2 \pi}
$$

A atenuação do canal pode então ser calculada através da equação 3 , onde $A$ é a área do fotorreceptor e $d$ a distancia entre os dispositivos.

$$
H(0)=R_{(\varphi)} \frac{A}{d^{2}} \cos \theta
$$

Finalmente podemos calcular a relação sinal ruido (SNR),

$$
S N R=\frac{R^{2} H^{2}(0) P_{t}^{2}}{\sigma_{\text {termico }}+\sigma_{\text {disparo }}},
$$

onde $\sigma_{\text {termico }}$ e $\sigma_{\text {disparo }}$ são, respectivamente, ruido de disparo e ruido térmico obtidos na equação 5 e na equação 6 .

$$
\sigma_{\text {disparo }}=2 q\left[R P_{r}\left(1+M_{I}^{2}\right)+I_{b g} I_{2}\right] B,
$$

onde $q$ é a carga do elétron, $M_{I}$ é o índice de modulação, $I_{b g}$ é a corrente de fundo, $I_{2}$ é o fator de ruido e $B$ é a largura de banda de ruido. Para esse trabalho foi o usado o padrão de modulação OOK (On Off Keying). O índice de modulação para o OOK é 0, 5.

$$
\sigma_{\text {termico }}=8 \pi k T_{k} \eta A B^{2}\left(\frac{I_{2}}{G}+\frac{2 \pi \Gamma}{g_{m}} \eta A I_{3} B\right),
$$

onde $k$ é a constante de Boltzmann, $T_{k}$ é a temperatura absoluta, $\eta$ é a capacitância fixa, $G$ é o ganho de tensão de malha aberta, $\Gamma$ o fator de ruido do canal e $I_{3}$ o ruido envolvendo o circuito [Kressel 2006].

\section{Modelagem do problema}

Este trabalho visa analisar o impacto do tamanho do pacote na eficiência do canal óptico sem fio. O modelo aqui proposto pode ser usado para determinar o tamanho de pacote para uma transmissão entre dois nós a partir da distância $d$ entre eles.

Consideremos então, dois dispositivos de comunicação VLC cada um dotado com um led para transmissão de dados e um fotorreceptor para recepção dos dados. Seja $E\left(d, T_{p}\right)$ a equação que determina a eficiência do uso do canal para a comunicação, determinar um tamanho de carga útil (payload) $T_{p}$ de maneira a maximizar $E$, para um valor de $d$ qualquer.

O modelo considera a comunicação entre dois nós. A transmissão de um pacote será considerado como correta quando não existir nenhum bit errado no pacote de dados e no pacote de confirmação (ACK). A figura 4 apresenta o diagrama para uma transmissão completa.

O IEEE 802.15.7 prevê como esquema de modulação o OOK, o VPPM (Variable Pulse Position Modulation) e o CSK (Color Shift Keying). Para esse trabalho considerou o padrão OOK, mas o mesmo pode ser aplicado para os outros dois padrões de modulação. 


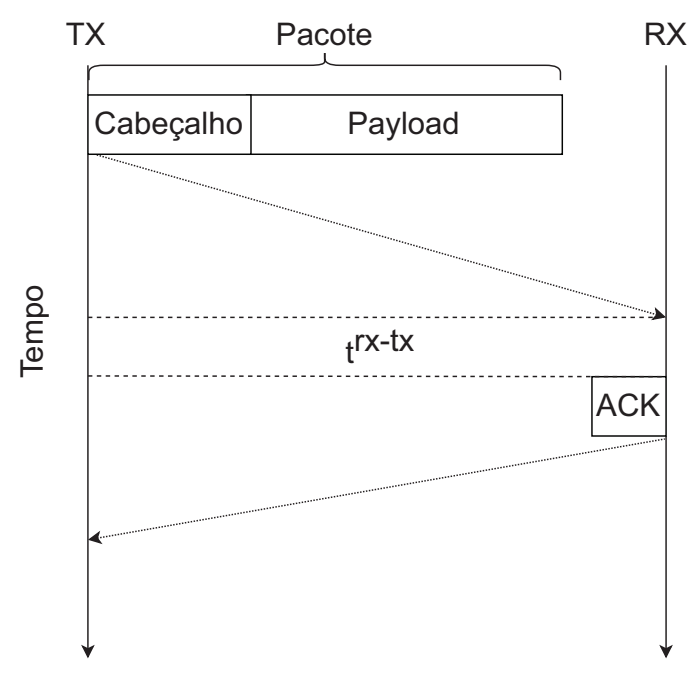

Figura 4. Diagrama para uma transmissão.

A taxa de erro de bits $B_{e}$ para a modulação OOK pode ser calculada através da equação 7 [Komine and Nakagawa 2004]

$$
B_{e}=Q(\sqrt{S N R})
$$

onde $Q(\sqrt{S N R})$ é a função-Q Marcum e pode ser calculada por

$$
Q(x)=\frac{1}{\sqrt{2 \pi}} \int_{x}^{\infty} e^{-y^{2} / 2} d y
$$

Com o valor obtido da taxa de erros, é possível então calcular a taxa de erro de pacotes $P_{e}$ (Equação 9). $N$ é o valor do total de bits transmitidos em um pacote dados somado com o total de bits em um pacote de confirmação.

$$
P_{e}=1-\left(1-B_{e}\right)^{N}
$$

Com a taxa de erro de pacotes calculada, é possível então calcular o número de pacotes que é necessário enviar para que um pacote seja enviado sem erros $P_{c}$. Esse valor pode ser obtido a partir da equação 10.

$$
P_{c}=\frac{1}{1-P_{e}}
$$

Finalmente a eficiência do canal $E\left(d, T_{p}\right)$ pode ser encontrada em função da distância e do tamanho de pacote (equação 11).

$$
E\left(d, T_{p}\right)=\frac{T_{p}}{P_{c} *\left[T_{p}+T_{c}+T_{A}+b *\left(2 * \frac{d}{c}+t^{r x-t x}\right)\right]},
$$


Sendo $T_{c}, T_{A}$ o tamanho do cabeçalho e o tamanho do ACK, respectivamente. A vazão é dada por $b, c$ é a velocidade da luz e $t^{r x-t x}$ é o tempo de processamento do pacote de dados.

\subsection{Tamanho ótimo do pacote VLC}

Para definir qual o tamanho ótimo do pacote deve-se maximizar a eficiência do canal. Para isso deve-se maximizar a equação 11. Ao fixar uma distância, é calculado a eficiência do canal para diversos tamanhos de payload.

Os valores dos parâmetros utilizados afim de calcular a atenuação do canal na equação 3 e o SNR na equação 4, são mostrados na tabela 1.

Tabela 1. Parâmetros para calculo SNR.

\begin{tabular}{c|c|c}
\hline Característica & Símbolo & Valor \\
\hline Área do fotorreceptor & $A$ & $5,24 \times 10^{-6} \mathrm{~m}^{2}$ \\
Responsividade do fotorreceptor & $R$ & $0,45 \mathrm{AW}$ \\
Potência transmitida & $P_{t}$ & $20 \times 10^{-3} \mathrm{~W}$ \\
Ângulo do receptor em relação ao emissor & $\varphi$ & $0^{\circ}$ \\
Ângulo de recepção & $\theta$ & $0^{\circ}$ \\
Corrente de fundo & $I_{b g}$ & $5100 \mu \mathrm{A}$ \\
Fator de Ruido & $I_{2}$ & 0,562 \\
Temperatura absoluta & $T_{k}$ & $297 \mathrm{~K}$ \\
Capacitância & $\eta$ & $112 \times 10^{-6} \mathrm{pF} / \mathrm{cm}^{2}$ \\
Ganho de Tensão & $G$ & 10 \\
Ruido do circuito & $I_{3}$ & 0,0868 \\
\hline
\end{tabular}

O padrão IEEE 802.15.7 específica todos os campos do cabeçalho para o OWC. Esse cabeçalho pode assumir diversos tamanhos dependendo do tipo do pacote, que pode ser do tipo beacon, do tipo dados, do tipo confirmação, do tipo comando, etc.

Para esse trabalho foram escolhidos três tamanhos de cabeçalho, sendo dois cabeçalhos especificados pela organização IEEE. O primeiro com o tamanho máximo definido no padrão, com 37 bytes de cabeçalho. Esse tamanho ocorre quando utiliza-se todos os campos e todos os campos usam seus respectivos tamanhos máximos. O segundo tamanho escolhido é o de 11 bytes, tal escolha se dá pelo fato de ele ser o menor cabeçalho de dados e ainda possuir os campos de endereçamento. O terceiro tamanho de cabeçalho escolhido foi de 9 bytes, o valor foi escolhido de forma que pudesse ser testado experimentalmente utilizando a plataforma OpenVLC [Wang et al. 2014].

Os parâmetros apresentados na tabela 2 foram utilizados na equação 11 para conseguir definir o tamanho ótimo do pacote de maneira a maximizar a eficiência do canal. As figuras 5, 6 e 7 mostram as curvas obtidas para a eficiência máxima do canal para os cabeçalhos de 37, 11 e 9 bytes respectivamente.

Todas as curvas crescem até um máximo. Esse valor máximo é o limite de eficiência que se pode obter para cada uma das distâncias. Nos pontos de máximo, para cada uma das curvas obtemos o valor de carga útil que maximiza a eficiência do canal. Esse valor de carga útil somado ao tamanho do cabeçalho chama-se tamanho ótimo do 
Tabela 2. Parâmetros para avaliação da eficiência do canal.

\begin{tabular}{c|c|c}
\hline Característica & Símbolo & Valor \\
\hline Carga útil & $T_{P}$ & variável (bits) \\
Numero de transmissões & $P_{c}$ & variável, depende da distância \\
Tamanho do cabeçalho & $T_{c}$ & variável $(296,88,72$ bits) \\
Tamanho do ACK & $T_{c}$ & 24 bits \\
Vazão & $b$ & $11.67 \mathrm{kbps}$ \\
Distância & $d$ & variável em metros \\
Velocidade da Luz & $c$ & $3 \times 10^{8} \mathrm{~m} / \mathrm{s}$ \\
\hline
\end{tabular}

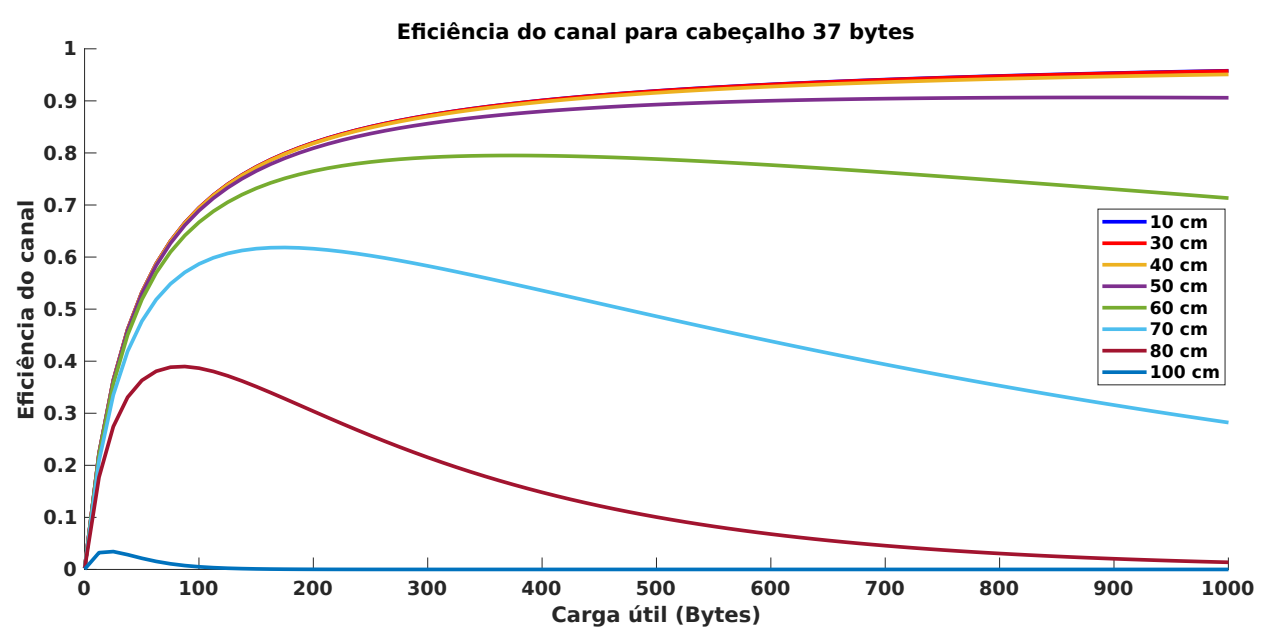

Figura 5. Eficiência do uso do canal para cabeçalho de 37 bytes.

pacote. Os valores a esquerda do máximo significa que está se transmitindo menos dados do que se poderia transmitir. Os valores a direita do máximo significa que estão sendo transmitidos mais dados, e, portanto, pacotes estão sendo perdidos que não estariam sendo perdidos.

Os resultados obtidos mostram que o tamanho do cabeçalho influencia diretamente na eficiência do uso do canal. Cabeçalhos maiores possuem tamanhos ótimos de pacotes menores e uma eficiência menor para uma mesma distância. Porém, a queda após o tamanho ótimo é menos acentuada. Isso se dá devido a relação entre o tamanho do payload em relação ao tamanho do cabeçalho ser maior.

\section{Avaliação experimental}

Para a avaliação experimental foi usado a plataforma OpenVLC 1.1. O OpenVLC é uma plataforma de comunicação por luz visível sem fio de baixo custo, de código aberto e definida por software. Ela funciona com uma plataforma de desenvolvimento, o BeagleBone Black (BBB). A BBB tem como sistema operacional uma distribuição Linux e funciona com a pilha de protocolos de rede Linux, podendo aproveitar também das ferramentas de rede desenvolvidas para o sistema operacional.

O software OpenVLC é implementado como um driver Linux e pode-se facilmente obter quando um pacote é enviado e recebido sem erros. Por ser uma plataforma 


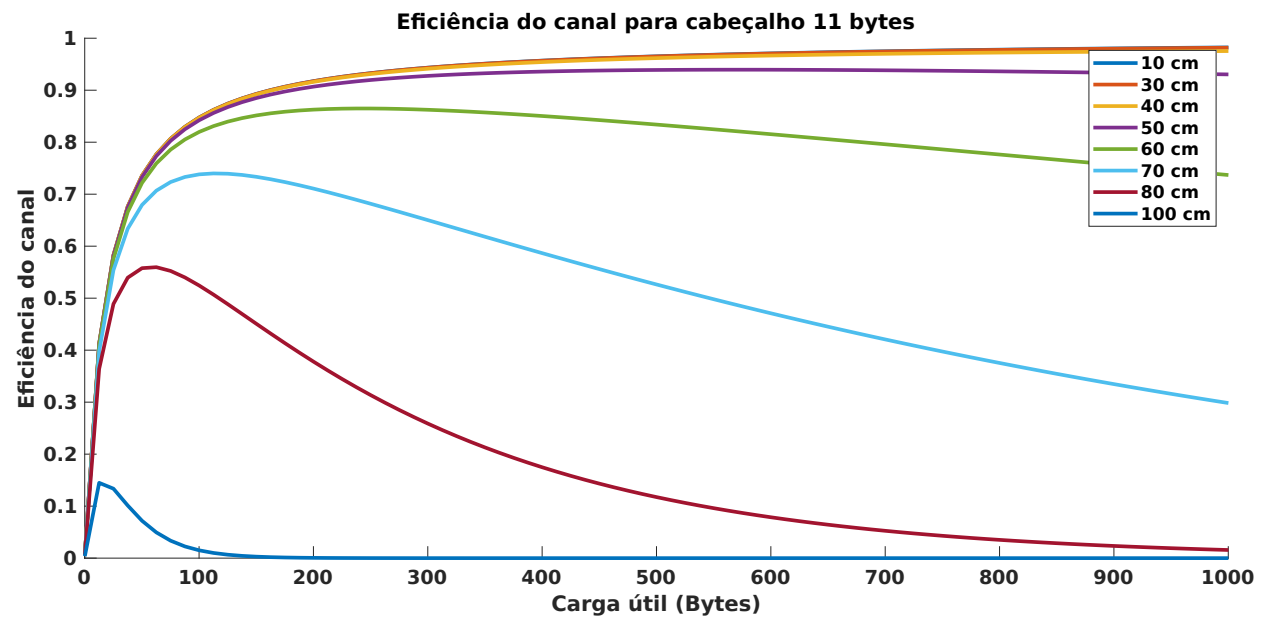

Figura 6. Eficiência do uso do canal para cabeçalho de 11 bytes.

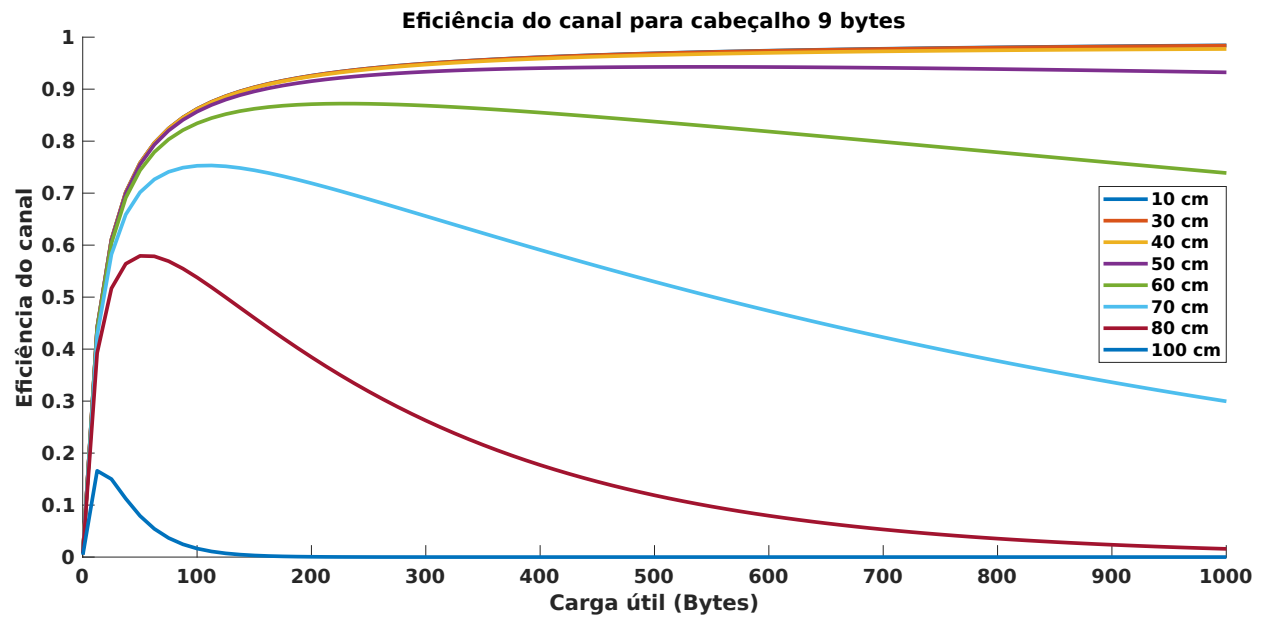

Figura 7. Eficiência do uso do canal para cabeçalho de 9 bytes. 


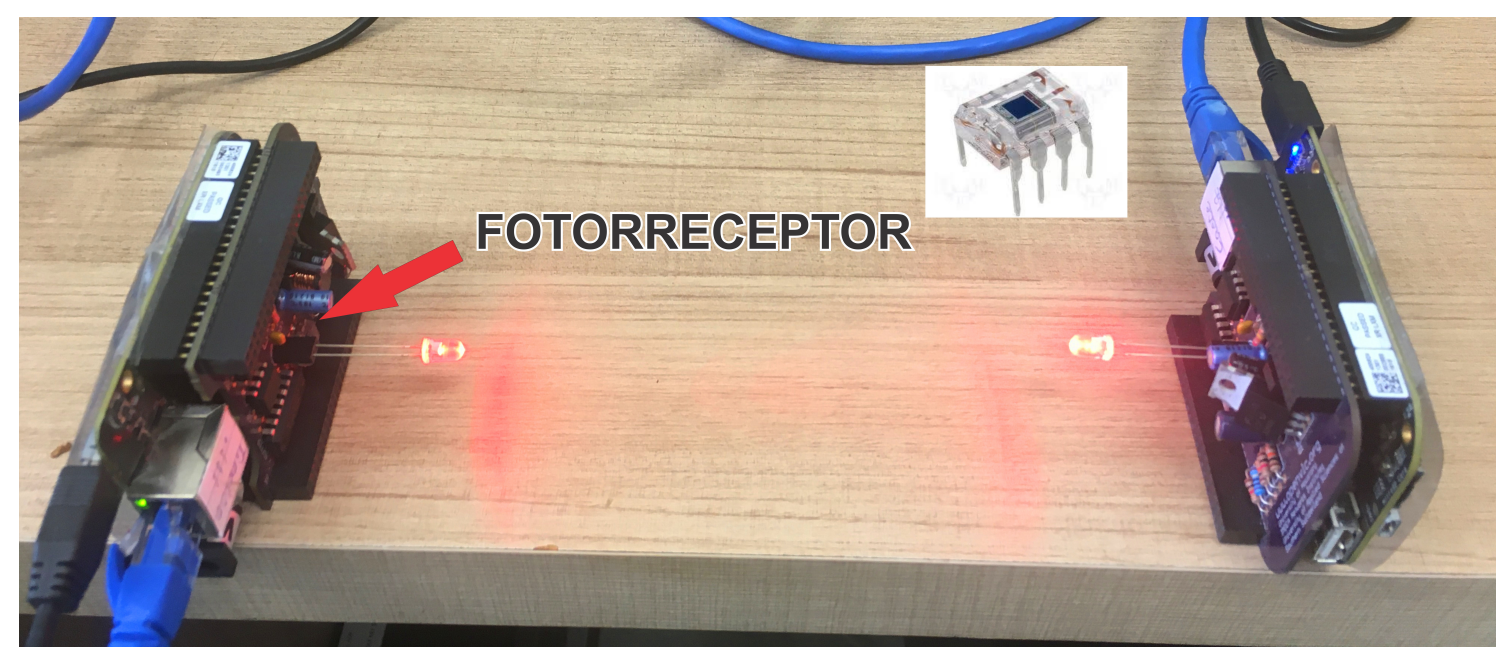

Figura 8. Ambiente experimental com dois BeagleBones e platafora OpenVLC.

definida por software pode-se editar vários parâmetros, entre eles o tamanho da unidade máxima de transmissão (MTU - do inglês Maximum Transmission Unit), possibilitando assim a realização dos experimentos.

A figura 8 mostra o ambiente experimental a seta em vermelho aponta o fotorreceptor. Foram utilizadas duas OpenVLC acopladas cada a uma BBB, ambas sobre uma mesa, de maneira que pudesse variar a distância entre elas. A iluminação no ambiente foi reduzida e controlada. Esse cuidado foi tomado para que não houvesse saturação do fotorreceptor e isso não se tornasse um fator que influenciasse nos resultados.

Com o número de pacotes enviados, o número de pacotes enviados corretamente e tamanho do payload em relação a quantidade de bits que são necessários para transmitir podemos calcular então qual a eficiência do uso do canal $E_{m}$ (equação 12)

$$
E_{m}=\frac{P_{e} c * \frac{T_{p}}{T_{p}+T_{c}+T_{A}}}{P_{s}}
$$

sendo $P_{e} c$ o número de pacotes enviados sem erros e $P_{s}$ o número total de pacotes enviados.

Foram realizados experimentos utilizando duas distâncias diferentes, $20 \mathrm{~cm}$ e 60 $\mathrm{cm}$. Para a distância de $20 \mathrm{~cm}$ foram utilizado 4 tamanhos de payload, 75 bytes, 500 bytes, 1000 bytes e 1500 bytes. Tais valores de tamanho de payload foram escolhidos de maneira ser o mais equidistante possível e de forma que descrevesse a curva da modelagem teórica.

Foram escolhidos 5 tamanhos diferentes de payload para realizar os experimentos a uma distância de $60 \mathrm{~cm}$. Os tamanhos utilizados foram 225 bytes, que é o tamanho ótimo de payload utilizando cabeçalho de 9 bytes e distância $60 \mathrm{~cm}$. Foram escolhidos também 2 tamanhos abaixo de 225 bytes, 75 e 150 bytes, e 2 valores acima, 300 e 375 bytes.

Para cada tamanho de pacote foram feitas 30 medições. Para cada medição, foram enviados ininterruptamente pacotes durante 150 segundos. Após os 150 segundos, eram medidos os números de pacotes enviados e o números de pacotes enviados sem erro. 
Tabela 3. Tamanho ótimo do payload e máxima eficiência de uso canal.

\begin{tabular}{|c|c|c|}
\hline Distância & Tamanho do payload & Eficiência do canal \\
\hline $0,2 \mathrm{~m}$ & 1863 bytes & 0,992 \\
$0,4 \mathrm{~m}$ & 1500 bytes & 0,978 \\
$0,6 \mathrm{~m}$ & 225 bytes & 0,872 \\
$0,8 \mathrm{~m}$ & 50 bytes & 0,580 \\
\hline
\end{tabular}

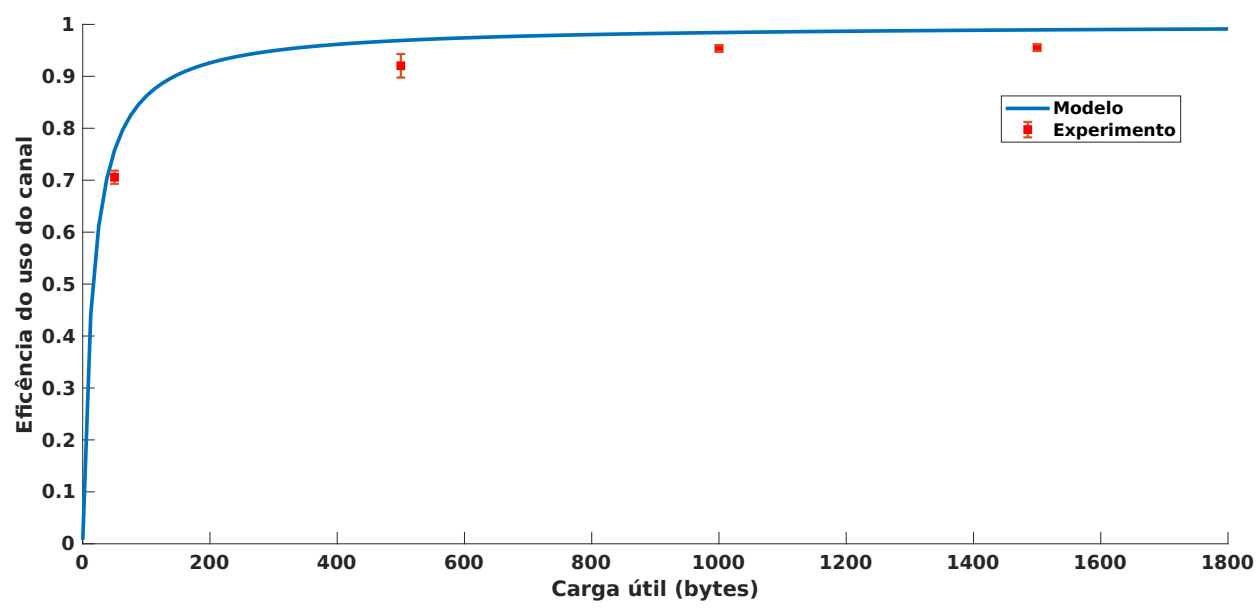

Figura 9. Eficiência de uso do canal para distância de $20 \mathrm{~cm}$.

A tabela 3 sumariza alguns valores de payload ótimos obtidos por meio do modelo proposto e qual é a máxima eficiência do uso do canal para uma determinada distância. $\mathrm{O}$ tamanho de 9 bytes de cabeçalho foi usado para construir a tabela. Por exemplo, pra uma distância de 0,4 m entre os nós, o tamanho de payload ótimo para maximizar a eficiência do canal é 1500 bytes e o máximo de eficiência do uso do canal é de $97,8 \%$.

A figura 9 mostra a eficiência do canal pelo tamanho de payload para uma distância de 0,2 m. A curva obtida a partir do modelo e os valores experimentais obtidos são mostrados. Foi aplicado então o método de correlação de Pearson [Grčar et al. 2005] de maneira a obter a correlação entre a curva obtida através do modelo e a curva dos valores experimentais. As duas curvas possuem uma tendencia de correlação de 0,998.

Já a figura 10 mostra a eficiência do uso do canal para uma distância de $60 \mathrm{~cm}$. O modelo prevê que para essa distância o valor que da carga útil que maximiza o uso do canal é de 225 bytes.A curva teórica e os valores experimentais obtidos possuem um correlação de 0,977. Valores de correlação entre 0,9 e 1 mostram uma correlação muito alta entre as curvas [Mukaka 2012].

Essa correlação alta mostra que os dados experimentais estão condizentes como os valores obtidos através do modelo. Ou seja, as duas curvas seguem a mesma tendência. De fato, ao observar nas figuras 9 e 10 as curvas teóricas e experimentais aparentam estar separadas por uma constante.

Como fatores para os dados experimentais não serem exatamente os valores calculados pelo modelo, podemos citar que ele é baseado que exista espaço livre entre o transmissor e o receptor. Portanto, o modelo não prevê ruídos de interferência devido a 


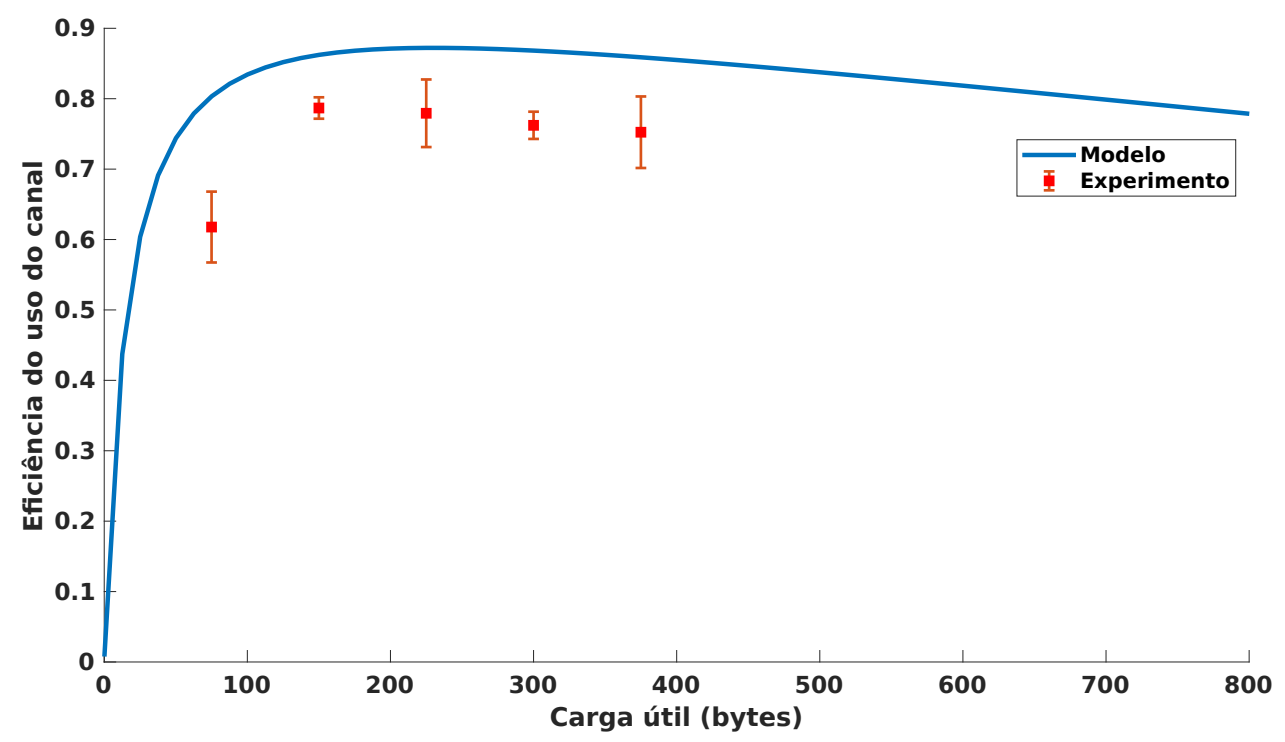

Figura 10. Eficiência de uso do canal para distância de $60 \mathrm{~cm}$.

reflexão do sinal. $\mathrm{O}$ aparato experimental foi montado em uma mesa de maneira tal que a luz emitida do emissor é refletida pela mesa e chega ao receptor com um certo atraso.

Outro ponto de destaque é que os dados de potência do led e responsividade do fotorreceptor que foram inseridos nas equações correspondem aos valores nominais disponibilizados pelos fabricantes. O OpenVLC não disponibiliza documentação de quanto de potência é fornecida ao led. Além disso para formulação do modelo foi assumido que o led emita, idealmente, obedecendo o modelo de emissão Lambertiano, leds reais podem sofrer variações sobre tal emissão.

\section{Conclusão e trabalhos futuros}

A OWC é uma solução viável para complementar a radiofrequência fornecendo uma solução para a escassez de recursos, oferecendo recursos necessários a um grande número de dispositivos. Transmitir dados de maneira eficiente e poupar recursos se torna essencial para diversas aplicações. Este artigo apresenta uma modelagem para se determinar o tamanho de pacote transmitido de maneira a maximizar a eficiência do uso do canal.

A partir dos dados experimentais é possível concluir que o modelo proposto para se determinar o tamanho ótimo do pacote para maximizar a eficiência do canal é bastante eficaz. O modelo mostra uma correlação com os dados experimentais muito alta chegando a até 0,998 .

Os trabalhos futuros incluem avaliar o modelo nos outros padrões de modulação que são descritos pelo IEEE 802.15.7. Também espera-se avaliar o modelo em cenários onde há vários nós se comunicando e em múltiplos saltos, podendo assim analisar qual o impacto que isso pode causar na eficiência de uso do canal.

\section{Referências}

Alliance, Z. (2004). Zigbee alliance finalizes specification. http://www . zigbee . org. [Online; acessado 25/03/2019]. 
Basnayaka, D. A. and Haas, H. (2015). Hybrid rf and vlc systems: Improving user data rate performance of vlc systems. In 2015 IEEE 81st Vehicular Technology Conference (VTC Spring), pages 1-5.

Cen, N., Jagannath, J., Moretti, S., Guan, Z., and Melodia, T. (2019). Lanet: Visible-light ad hoc networks. Ad Hoc Networks, 84:107-123.

CISCO (2019). Internet of Things. https://www.cisco.com/c/ $\mathrm{dam} / \mathrm{en} / \mathrm{us} / \mathrm{products} / \mathrm{collateral} / \mathrm{se} /$ internet-of-things/ at-a-glance-c45-731471.pdf. [Online; access November-27-2019].

Grčar, M., Mladenič, D., Fortuna, B., and Grobelnik, M. (2005). Data sparsity issues in the collaborative filtering framework. In International Workshop on Knowledge Discovery on the Web, pages 58-76. Springer.

Hassan, N. U., Naeem, A., Pasha, M. A., Jadoon, T., and Yuen, C. (2015). Indoor positioning using visible led lights: A survey. ACM Comput. Surv., 48(2):20:1-20:32.

IEEE (2011). Ieee standard for local and metropolitan area networks-part 15.7: Shortrange wireless optical communication using visible light. IEEE Std 802.15.7-2011, pages $1-309$.

IEEE (2019). Ieee standard for local and metropolitan area networks-part 15.7: Shortrange optical wireless communications. IEEE Std 802.15.7-2018 (Revision of IEEE Std 802.15.7-2011), pages 1-407.

Jagannath, J. and Melodia, T. (2019). Vl-route: A cross-layer routing protocol for visible light ad hoc network. arXiv preprint arXiv:1904.05177.

Jun Yin, Xiaodong Wang, and Agrawal, D. P. (2004). Optimal packet size in error-prone channel for ieee 802.11 distributed coordination function. In 2004 IEEE Wireless Communications and Networking Conference (IEEE Cat. No.04TH8733), volume 3, pages 1654-1659 Vol.3.

Kavehrad, M. and Zhang, W. (2015). Light positioning system (LPS). Cambridge University Press.

Komine, T. and Nakagawa, M. (2004). Fundamental analysis for visible-light communication system using led lights. IEEE Transactions on Consumer Electronics, 50(1):100-107.

Kressel, H. (2006). Semiconductor devices for optical communication, volume 39. Springer Science \& Business Media.

Matheus, L. E. M. et al. (2018). Dyrp-vlc: a dynamic routing protocol for visible light communication networks.

Matheus, L. E. M., Vieira, A. B., Freire, J. H. F., Vieira, L. F. M., Vieira, M. A. M., and Gnawali, O. (2017). Comunicação por Luz Visível: Conceitos, Aplicações e Desafios. Minicurso do Simpósio Brasileiro de Redes de Computadores e Sistemas Distribuídos 2017.

Matheus, L. E. M., Vieira, A. B., Vieira, L. F. M., Vieira, M. A. M., and Gnawali, O. (2019). Visible light communication: Concepts, applications and challenges. IEEE Communications Surveys Tutorials, pages 1-1. 
Mukaka, M. (2012). Statistics corner: a guide to appropriate use of correlation in medical research. Malawi Medical Journal, 24(3):69-71.

Pathak, P. H., Feng, X., Hu, P., and Mohapatra, P. (2015). Visible light communication, networking, and sensing: A survey, potential and challenges. IEEE Communications Surveys Tutorials, 17(4):2047-2077.

Pompili, D., Melodia, T., and Akyildiz, I. F. (2006). Routing algorithms for delayinsensitive and delay-sensitive applications in underwater sensor networks. In Proceedings of the 12th Annual International Conference on Mobile Computing and Networking, MobiCom '06, pages 298-309, New York, NY, USA. ACM.

Sankarasubramaniam, Y., Akyildiz, I. F., and McLaughlin, S. W. (2003). Energy efficiency based packet size optimization in wireless sensor networks. In Proceedings of the First IEEE International Workshop on Sensor Network Protocols and Applications, 2003., pages $1-8$.

Shao, S., Khreishah, A., Rahaim, M. B., Elgala, H., Ayyash, M., Little, T. D. C., and $\mathrm{Wu}, \mathrm{J}$. (2014). An indoor hybrid wifi-vlc internet access system. In 2014 IEEE 11th International Conference on Mobile Ad Hoc and Sensor Systems, pages 569-574.

Soares Junior, W. A. S. and Vieira, L. F. M. (2014). Redes aéreas ieee 802.15.4: tamanho ótimo do pacote e coleta de dados.

Ucar, S., Coleri Ergen, S., Ozkasap, O., Tsonev, D., and Burchardt, H. (2016). Secvlc: Secure visible light communication for military vehicular networks. In Proceedings of the 14th ACM International Symposium on Mobility Management and Wireless Access, MobiWac '16, pages 123-129, New York, NY, USA. ACM.

Vieira, A. G., Vieira, L. F. M., Veira, M. A. M., and Vieira., A. B. (2018). Comunicação por luz visível: redução da latência via combinação de pacotes. In SBESC 2018 ().

Vries, J. P. D., Simić, L., Achtzehn, A., Petrova, M., and Mähönen, P. (2014). The wifi "congestion crisis": Regulatory criteria for assessing spectrum congestion claims. Telecommunications Policy, 38(8):838 - 850. Special issue on Moving Forward with Future Technologies: Opening a Platform for All Special issue on Papers from the 41st Research Conference on Communication, Information and Internet Policy (TPRC 2013).

Wang, Q., Giustiniano, D., and Puccinelli, D. (2014). Openvlc: Software-defined visible light embedded networks. In Proceedings of the 1st ACM MobiCom workshop on Visible light communication systems, pages 15-20. ACM.

Zhong, W. and Wang, Z. (2015). Performance enhancement techniques for indoor vlc systems. In Visible light Communication. Cambridge University Press.

Zuquim, A. L. A., Vieira, L. F. M., Vieira, M. A., Vieira, A. B., Carvalho, H. S., Nacif, J. A., Coelho, C., da Silva, D., Fernandes, A. O., and Loureiro, A. A. F. (2003). Efficient power management in real-time embedded systems. In Emerging Technologies and Factory Automation, 2003. Proceedings. ETFA'03. IEEE Conference, volume 1, pages 496-505. IEEE. 